\title{
A combined model for short-term wind speed forecasting based on empirical mode decomposition, feature selection, support vector regression and cross-validated lasso
}

\author{
Tao Wang ${ }^{\text {Corresp. } 1}$ \\ ${ }^{1}$ Hefei University of Technology, Hefei, China \\ Corresponding Author: Tao Wang \\ Email address: wtustc@mail.ustc.edu.cn
}

Background: The planning and control of wind power production rely heavily on short-term wind speed forecasting. Due to the non-linearity and non-stationarity of wind, it is difficult to carry out accurate modeling and prediction through traditional wind speed forecasting models. Methods: In the paper, we combine empirical mode decomposition (EMD), feature selection (FS), support vector regression (SVR) and cross-validated lasso (LassoCV) to develop a new wind speed forecasting model, aiming to improve the prediction performance of wind speed. EMD is used to extract the intrinsic mode functions (IMFs) from the original wind speed time series to eliminate the non-stationarity in the time series. FS and SVR are combined to predict the high-frequency IMF obtained by EMD. LassoCV is used to complete the prediction of low-frequency IMF and trend. Results: Data collected from two wind stations in Michigan, USA are adopted to test the proposed combined model. Experimental results show that in multi-step wind speed forecasting, compared with the classic individual and traditional EMD-based combined models, the proposed model has better prediction performance. Conclusions: Through the proposed combined model, the wind speed forecast can be effectively improved. 
1 A combined model for short-term wind speed

2 forecasting based on empirical mode decomposition,

3 feature selection, support vector regression and cross-

4 validated lasso

5

6 Tao Wang ${ }^{1}$

7

$8{ }^{1}$ School of Computer and Information, Hefei University of Technology, Hefei, Anhui, China 9

10 Corresponding Author:

11 Tao Wang ${ }^{1}$

12 Fuicui Lake Campus of HeFei University of Technology, Hefei, Anhui, China

13 Email address: wtustc@mail.ustc.edu.cn (T. Wang) 


\section{Abstract}

16 Background: The planning and control of wind power production rely heavily on short-term wind 17 speed forecasting. Due to the non-linearity and non-stationarity of wind, it is difficult to carry out 18 accurate modeling and prediction through traditional wind speed forecasting models.

19 Methods: In the paper, we combine empirical mode decomposition (EMD), feature selection (FS), support vector regression (SVR) and cross-validated lasso (LassoCV) to develop a new wind speed forecasting model, aiming to improve the prediction performance of wind speed. EMD is used to extract the intrinsic mode functions (IMFs) from the original wind speed time series to eliminate the non-stationarity in the time series. FS and SVR are combined to predict the high-frequency IMF obtained by EMD. LassoCV is used to complete the prediction of low-frequency IMF and trend.

Results: Data collected from two wind stations in Michigan, USA are adopted to test the proposed combined model. Experimental results show that in multi-step wind speed forecasting, compared with the classic individual and traditional EMD-based combined models, the proposed model has better prediction performance.

30 Conclusions: Through the proposed combined model, the wind speed forecast can be effectively improved.

\section{Introduction}

As a sustainable and renewable energy alternative to traditional fossil fuels, wind power has attracted widespread attention and rapid development in recent years (Hu et al. 2018). According to the statistical report of the Global Wind Energy Council, the world capacity is about $650.8 \mathrm{GW}$ (Fu et al. 2020), of which the installed capacity in 2019 is 59.7 GW (GLOBAL 2020). However, with the increase of grid-connected wind power, the stability of the power system will be challenged (Liu et al. 2018a). This is because wind power is closely related to the non-stationarity of wind speed. Accurate wind speed forecasting will provide support for wind power planning and control, and even help reduce the impact of unexpected events on the stability of the power system (Liu et al. 2018b). But due to the non-linearity and non-stationarity of wind, it is difficult to establish a satisfactory wind speed forecasting model. To this end, researchers have made great efforts to improve forecasting performance from different aspects, including basic predictive models, preprocessing methods, and combined or hybrid strategies.

For basic predictive models, a variety of methods has been presented, mainly including physical models, statistical models, and machine learning. The physical model usually uses physical parameters such as temperature and pressure to predict wind speed (Heng et al. 2016). Numerical 
49 correlation between physical parameters and short-term wind speed, this type of model can only 50 be used for medium- and long-term wind speed forecasting, not for short-term wind speed 51 forecasting. In the short-term wind speed forecasting, the wind speed is generally predicted by 52 analyzing the inherent laws of historical wind speed data (Chen et al. 2018; Liu et al. 2018b).

53 The statistical model is a method widely used in short-term wind speed forecasting, which uses 54 historical data to predict wind speed. Commonly used statistical models have autoregressive (AR) 55 (Lydia et al. 2016a), autoregressive moving average (ARMA) (Torres et al. 2005) and 56 autoregressive integrated moving average (ARIMA) (Wang \& Hu 2015). Kavasseri et al. 57 (Kavasseri \& Seetharaman 2009) proposed an f-ARIMA model for wind speed forecasting, and 58 claimed that compared with the persistence model, their model has significantly improved the 59 prediction accuracy. Maatallah et al. (Ait Maatallah et al. 2015) developed a Hammerstein 60 autoregressive model to predict wind speed, and verified that their model has a better root mean 61 square error (RMSE) than ARIMA and ANN. Poggi et al. (Poggi et al. 2003) developed a model 62 to predict wind speeds of three Mediterranean sites in Corsica based on AR, and proved that the 63 synthetic time series can retain the statistical characteristics of wind speeds. Likely, Lydia et al. 64 (Lydia et al. 2016b) presented a short-term wind speed forecasting model by combining linear AR 65 and non-linear AR. In general, the statistical model is based on the linear assumption of data, while 66 the wind speed series have non-linear characteristics, which makes those methods unable to 67 effectively deal with the non-linear characteristics of wind.

68 To solve the problem, machine learning is introduced by researchers to predict wind speed. 69 Normally, machine learning is used as a predictive model or parameter optimization, mainly 70 includes the evolutionary algorithm, extreme learning machine (ELM) algorithm, ANN algorithm 71 and SVM algorithm. Wang et al. (Wang 2017) presented a wind speed forecasting model by 72 combining SVM and particle swarm optimization (PSO). Zhang et al. (Zhang et al. 2019) 73 combined online sequential outlier robust ELM with hybrid mode decomposition (HMD) to 74 predict wind speed. Wang et al. (Wang et al. 2018) developed an error correction-based ELM 75 model for short-term wind speed forecasting. Liu et al. (Liu et al. 2020) introduced the Jaya-SVM 76 (Jaya algorithm-based support vector machine) into wind speed forecasting. Krishnaveny et al. 77 (Nair et al. 2017) exploited the performance of three different models, i.e. ANN, ARIMA and 78 hybrid model, in wind speed forecasting. Azeem et al. (Azeem et al. 2018) investigated the KNN79 based and ANN-based models for wind speed forecasting. Recently, deep learning, a new branch 80 of machine learning, has received extensive attention. It has been widely used for regression and 81 classification problems. According to the literature, deep learning can abstract the hidden structure 82 and inherent characteristics of data compared with shallow methods. Khodayar et al. (Khodayar \& 
83 Wang 2019) introduced a scalable graph convolutional deep learning (GCDLA) for wind speed

84 forecasting. Wang et al. (Wang et al. 2016a) investigated a deep belief network model for wind 85 speed forecasting. Khdayar et al. (Khodayar et al. 2019) combined rough set theory and restricted 86 Boltzmann machines presented a wind speed forecasting. Hong et al. (Hong \& Satriani 2020) 87 based on a convolutional neural network developed a day-ahead wind speed forecasting model. 88 Although researchers claim that deep learning can achieve better performance, these methods are 89 computationally intensive and prone to overfitting on small data sets.

90 In addition to these basic forecasting models, preprocessing methods such as feature selection (FS) 91 are also introduced in wind speed forecasting. This is because in short-term wind speed 92 forecasting, the lag of historical wind speed is usually used as the feature, which may lead to a 93 certain degree of redundancy. FS is used to select the best input for the basic predictive model, so 94 that the model can obtain better generalization performance ( $\mathrm{Li}$ et al. 2018a). For example: 95 Paramasivan et al. (Paramasivan \& Lopez 2016) employed a ReliefF feature selection algorithm 96 to identify key features, and then used a bagging neural network to predict the wind speed. Niu et 97 al. (Niu et al. 2018) presented a multi-step wind speed forecasting model using optimal FS, 98 modified bat algorithm and cognition strategy. Botha et al. (Botha \& Walt 2017) combined FS 99 with SVM to predict short-term wind speed. Kong et al. (Kong et al. 2015) combined feature 100 selection and reduced support vector machines (RSVM) for wind speed forecasting.

101 Due to the unstable nature of wind, the model of combined- or hybrid-signal processing technology 102 has become the mainstream of wind speed forecasting. Wherein the signal processing technology 103 is usually employed to decompose the wind speed to reduce or eliminate the instability. Commonly 104 used signal processing techniques have empirical mode decomposition (EMD), variational mode 105 decomposition (VMD) and wavelet transform (WT). Wang et al. (Wang et al. 2016b) decomposed 106 wind speed into stable signals using ensemble empirical mode decomposition (EEMD). Sun et al. 107 (Sun \& Wang 2018) developed a fast ensemble empirical mode decomposition model to improve 108 the accuracy of wind speed forecasting. Tascikaraoglu et al. (Tascikaraoglu et al. 2016) based on 109 WT proposed a wind speed forecasting model. Hu et al. (Hu \& Wang 2015) adopted an empirical 110 wavelet transform (EWT) to extract key information in wind speed time series. Yu et al. (Yu et al. 111 2017) explored the performance of EMD, EEMD and complete ensemble empirical mode 112 decomposition with adaptive noise (CEEMDAN) in wind speed forecasting.

113 In the field of wind speed forecasting, there are mainly three forecast scenarios: short-term 114 forecasting, medium-term forecasting and long-term forecasting. Among them, short-term wind 115 speed forecasting is essential for estimating power generation, and it is difficult to predict 116 accurately due to the nonlinearity and instability of wind speed. Therefore, in the study, we tried 
117 to develop a new model to forecast short-term wind speed. The originality of this model is to 118 propose a combined model of EMD, FS, SVR and Cross-validated Lasso (LassoCV) for multi-

119 step wind speed forecasting. The framework of our study is as follows: (a) EMD is used to extract

120 the intrinsic mode functions (IMFs) from the original wind speed time series; (b) FS and SVR are

121 combined to predict high-frequency IMF; (c) LassoCV is used to complete the prediction of low-

122 frequency IMF and trend.

123 The main contributions of the research are as follows:

124 (1) A novel model based on EMD, FS, SVR and LassoCV is proposed to improve the accuracy

125 of multi-step wind speed forecasting, where EMD is used to extract IMFs from the original

126 wind speed data to reduce the non-stationarity of wind speed.

127 (2) Based on the principle of EMD, the first IMF component decomposed by EMD contains most

128 of the high-frequency information, and an algorithm with good generalization performance is

129 usually required for prediction. We combine FS and SVR to predict the high-frequency IMF

130 (i.e., the first IMF) component.

131 (3) Compared with the first IMF component, the frequency of the other IMF components

132 decomposed by EMD is much lower and presents a Sin-like curve. Linear regression usually

133 gets better performance. We introduce LassoCV to complete the prediction of low-frequency

$134 \quad$ IMFs and trend.

135 The paper is as follows: The framework of the proposed model and the principles involved are

136 introduced in Section 2. Section 3 describes the experimental data used in the paper, and the

137 comparison with the classic individual models. Section 4 discusses the effectiveness of EMD.

138 Section 5 concludes the study.

139 Methods

140 The whole process of the proposed model

141 The architecture of our proposed model is shown in Fig. 1. The whole process is as follows:

142 (1) Use EMD to decompose wind speed into a series of IMFs. EMD algorithm is introduced in 143 Section 2.2.

144 (2) Combine FS and SVR to predict the high-frequency IMF obtained by EMD. FS and SVR algorithms are provided in Section 2.3 and Section 2.4, respectively.

146 (3) Use LassoCV to complete the prediction of the low-frequency IMF and trend. LassoCV algorithm is listed in Section 2.5.

148 (4) Performance evaluation. The performance indicators are introduced in Section 2.6, and the experimental results and analysis are given in Sections 3 and 4. 


\section{Empirical model decomposition}

151 Due to the non-stationarity, intermittent and inherent nature of wind speed, it is difficult to directly

152 predict the future wind speed. One possible solution is to decompose different frequencies from

153 chaotic wind data (Bokde et al. 2019) and use models to predict them separately. Based on this

154 idea, the study introduces signal processing technology to decompose wind speed. Common signal

155 decomposition algorithms include Wavelet transform, morphology filters, EMD and many others.

156 Wavelet transform is not adaptive and follows the prior knowledge of its mother wavelet, so

157 somewhat limits its ability to extract nonlinear and non-stationary components from the data.

158 Similarly, the morphology filters have to select the shape and the length of the structural element.

159 There is no uniform standard and depends on human experience, whereas EMD has received great

160 attention from researchers because of its superior performance and easy-to-understand. Therefore,

161 in this study, we use EMD to preprocessing the wind speed.

162 EMD is essentially a non-linear signal analysis method that can handle non-linear and non-

163 stationary time series (Huang et al. 1998). EMD uses the time-scale characteristics of the data to

164 decompose the signal, and does not need to set any basis functions in advance. In theory, EMD

165 can be applied to any type of signal. Since EMD was proposed, it has been rapidly applied to many

166 different engineering fields such as marine and atmospheric research, seismic record analysis and

167 mechanical fault diagnosis (Gao \& Liu 2021).

168 The basic idea of EMD is to decompose non-stationary time series signals into a series of IMFs

169 along with a residue (Huang et al. 1998). The IMF should meet two principles: (1) the number of

170 extreme and zero values must be equal or differ by at most one; (2) the average value of upper

171 envelop and lower envelope must be zero (Ziqiang \& Puthusserypady 2007). Let $s(t), t=1,2, \ldots, l$

172 be a time series. EMD decomposition steps are as follows:

173 Step 1: Identify the local minima and maxima of the time series.

174 Step 2: Use cubic splines to interpolate local minima and maxima values to generate lower $s_{l}$

$175(t)$ and upper $s_{u}(t)$.

176 Step 3: Computer the average envelope of the upper and lower envelopes

$$
m_{t}=\frac{s_{u}(t)+s_{l}(t)}{2}
$$

Step 4: Subtract the average envelope from the original time series $h(t)=s(t)-m_{t}$

179 Step 5: Check $h(t)$ if meets the two principles of IMF. If so, treat $h(t)$ as the new IMF $c(t)$ and 180 calculate the residual signal $r(t)=s(t)-h(t)$. Otherwise, replace $h(t)$ with $s(t)$, and then repeat 181 steps 1 to 5.

182 Step 6: Set $r(t)$ as new $s(t)$ and repeat steps 1 to 5 until all IMFs are obtained. 
183 Through the whole process, a set of IMFs from high to low frequency can be extracted from the 184 time series. Therefore, the original time series can be expressed as:

$$
s(t)=\sum_{i=1}^{n} c_{i}(t)+r_{n}(t)
$$

186

where $n$ is the number of IMFs. $c_{i}(t)$ refers to the IMF, which is periodic and almost orthogonal to each other ( $\mathrm{Li}$ et al. 2018b). $r_{n}(t)$ is the final residual representing the trend of $s(t)$.

\section{Feature selection}

After obtaining the IMF components of wind speed, we need to predict it. In the study, we use the observed and lag of the IMF components as the raw features, respectively forecast each IMF component, and add all the predicted IMF components to get the final wind speed. Despite, the raw features contain sufficient information for forecasting, some irrelevant or partially relevant features in the raw features may have a negative impact on the model. To avoid the impact, a selection algorithms include filter method, wrapper method, heuristic search algorithm, embedded method (Chandrashekar \& Sahin 2014). In this study, we use the filter method. In order to obtain scores of different variables, we use the univariate linear regression test to calculate the correlation between features and output (Liu et al. 2019b), which is defined as :

$$
\operatorname{Cor}_{i}=\frac{(X[:, i]-\operatorname{mean}(X[:, i])) *(y-\text { mean }(y))}{\operatorname{std}(X[:, i]) * \operatorname{std}(y)}
$$

where $X$ is an $N \times M$ matrix, each column is a feature. $y$ is the $N \times 1$ vector of the output we are interested in. Based on the rank of correlation, the irrelevant or partially relevant features are removed.

\section{Support vector regression}

The support vector machine (SVM) is a learning method based on structural risk minimization criteria, which can minimize the expected risk and obtain better generalization performance on unknown data. The support vector regression (SVR) is an extension of SVM for regression problems (Drucker et al. 1997). Due to the nonlinear and non-stationary nature of wind speed, SVR is widely used in short-term wind speed forecasting (Khosravi et al. 2018; Liu et al. 2019a; Santamaría-Bonfil et al. 2016). In the research, we use EMD to decompose the IMF components of wind speed, and the high-frequency IMF component contains the nonlinear and non-stationary

211 part of wind speed. In order to obtain better generalization performance, we refer to existing 212 research and use SVR to predict it.

213 The main idea of SVR is to implement linear regression in the high-dimensional feature space 
214 obtained by mapping the original input through a predefined function $\varnothing(x)$, and to minimize 215 structure risks (Chen et al. 2018). Given a set of samples $\left\{x_{i}, y_{i}\right\}, i=1,2, \ldots, N, y_{i}$ is the output and $x_{i}$ 216 is the input. The objective is:

$$
\begin{gathered}
f(x)=W^{T} \emptyset(x)+b \\
R[f]=\frac{1}{2}\|W\|^{2}+C \sum_{i=1}^{N} L\left(x_{i}, y_{i}, f\left(x_{i}\right)\right)
\end{gathered}
$$

where $W$ and $b$ are the regression coefficient and bias, respectively. $C$ is the penalty coefficient. $L$ $\left(x_{i}, y_{i}, f\left(x_{i}\right)\right)$ represents the loss function, and $R[f]$ is the structure risk. The corresponding constrained optimization problem can be expressed as:

$$
\begin{array}{cc}
\min & \frac{1}{2}\|W\|^{2}+C \sum_{i=1}^{N}\left(\xi_{i}+\xi_{i}^{*}\right) \\
\text { s.t. } \quad & y_{i}-W^{T} \phi(x)-b \leq \epsilon+\xi_{i} \\
& W^{T} \phi(x)+b-y_{i} \leq \epsilon+\xi_{i}^{*} \\
& \xi_{i}, \xi_{i}^{*} \geq 0, i=1,2, \ldots, n
\end{array}
$$

222

where $\xi_{i}$ and $\xi_{i}^{*}$ refer to the slack variables. By introducing the Lagrange multiplier, the regression can be expressed as:

where $\alpha_{i}$ and $\alpha_{i}^{*}$ are the Lagrange multipliers that satisfy the conditions $\alpha_{i} \geq 0, \alpha_{i}^{*} \geq 0$ and $\sum_{i=1}^{N}\left(\alpha_{i}-\alpha_{i}^{*}\right)=0 . K\left(x_{i}, x\right)$ is the kernel function conforming to Mercer's theorem.

\section{Cross-validated lasso}

The Lasso algorithm is a regression model that can perform feature selection and regularization at the same time. It was originally proposed by Robert Tibshirani of Stanford University, with better prediction accuracy and interpretability (Tibshirani 1996). Normally, in regression, we want to find a coefficient $\beta=\left(\beta_{1}, \ldots, \beta_{p}\right)$ that satisfies the following:

$$
f(x)=\sum_{i=1}^{N}\left(\alpha_{i}-\alpha_{i}^{*}\right) K\left(x_{i}, x\right)+b
$$




$$
\min _{\beta_{0}, \beta}\left\{\frac{1}{N} \sum_{i=1}^{N}\left(y_{i}-\beta_{0}-x_{i}^{T} \beta\right)^{2}\right\}
$$

$$
\text { s.t. } \sum_{j=1}^{p}\left|\beta_{j}\right| \leq t
$$

237

238

Rewritten in the Lagrangian form:

$$
\hat{\beta}_{\text {lasso }}=\underset{\beta \in R^{p}}{\operatorname{argmin}}\left\{\frac{1}{N}\|y-X \beta\|_{2}^{2}+\lambda\|\beta\|_{1}\right\}
$$

The $L_{1}$-norm is used instead of the $L_{2}$-norm in Lasso. Since the constraint region is diamondshaped, it is more likely to pick the solution that lies at the corner of the region. As a result, the solution of the lasso is sparse, with some coefficients set to exactly equal to zero, that is, Lasso performs a straightforward feature selection.

To estimate $\hat{\beta}_{\text {lasso }}$, the value of the penalty parameter $\lambda$ is critically important. However, the optimal $\lambda$ is not given automatically. If $\lambda$ is chosen appropriately, Lasso achieves the fast convergence under fairly general conditions; On the other hand (chosen inappropriately), Lasso may be inconsistent or have a slower convergence. In the paper, we adopt the cross-validated Lasso algorithm, in which the penalty parameter $\lambda$ is chosen based on cross-validation, and this is also the leading recommendation way in the theoretical literature (Park \& Casella 2008).

\section{Prediction performance criteria}

In the study, the mean absolute percentage error (MAPE), mean absolute error (MAE) and RMSE are used as performance indicators to evaluate the proposed wind forecasting model, which are defined as follows:

$$
\begin{gathered}
\text { MAPE }=\frac{1}{N} \sum_{i=1}^{N}\left|\left(Y_{i}-\hat{Y}_{i}\right) / Y_{i}\right| \\
M A E=\frac{1}{N} \sum_{i=1}^{N}\left|Y_{i}-\hat{Y}_{i}\right| \\
R M S E=\sqrt{\frac{1}{N-1} \sum_{\mathrm{i}=1}^{N}\left(Y_{i}-\hat{Y}_{i}\right)^{2}}
\end{gathered}
$$

where $Y_{i}$ and $\hat{Y}_{i}$ refer to the observed and predicted wind speed of data point $i$, respectively. For 257 
258 Results

259

260

261

262

263

264

265

266

267

268

269

270

271

272

273

274

275

276

277

278

279

280

281

282

283

284

285

286

287

288

289

290

\section{Wind speed data}

The wind speed data used in the study is gathered from two wind stations in Michigan, USA from September 2019 to October 2019. The number of data is 1,464. The initial 50 days from September 1, 2019 to October 20, 2019 are employed as input for model training, and the remaining days, i.e., from October 21, 2019 to October 31, 2019 are used to test. Fig. 2 shows these two wind speed time series, and the corresponding statistics are listed in Table 1.

\section{Experiments and result analysis}

To verify the effectiveness of the proposed model, we compare it with five classic individual models, including Persistence, ELM, SVR and ANN, ARIMA. The 1- to 3-step forecasting results of these models under time series $\# 1$ and $\# 2$ are displayed in Fig. 3-4, and the corresponding error estimated results are listed in Table 2-5. It is worth noting that for a fair comparison, the parameters of the involved models are selected based on cross-validation. Based on the experimental results, we can get the following conclusions:

(1) In the 1-step forecasting, for wind station \#1, the proposed model obtains the best accuracy: RMSE, MAE, and MAPE are 0.5859, 0.4426, and 21.11\%, respectively. The classic individual models from low to high based on RMSE are ELM, ANN, Persistence, SVR, and ARIMA, with MAPE values of $36.20 \%, 36.24 \%, 36.20 \%, 34.87 \%$, and $34.25 \%$, respectively. Likely, in wind station \#2, compared with the classic individual models, the proposed model still obtains the best performance, and the MAPE value is $17.10 \%$.

(2) In the 2-step forecasting, when wind station \#1 is used, the proposed model has the lowest performance criteria, i.e., the values of RMSE, MAE, and MAPE are 0.7531, 0.5848, and $24.78 \%$, respectively. In addition, for wind station \#2, the proposed model still achieves the lowest performance criteria value. Take MAPE as an example, the value of MAPE is $22.99 \%$, which is significantly lower than other models.

(3) In the 3-step forecasting, the proposed model is still the model with the highest prediction accuracy, and the MAPE of wind stations $\# 1$ and $\# 2$ are $27.55 \%$ and $24.59 \%$, respectively. And Persistence has the worst RMSE value among these models, with MAPE of 57.64\% and $47.99 \%$, respectively.

In general, under 1- to 3-step forecasting, the proposed model can obtain the best prediction performance compared with the classic individual models.

\section{Compared with traditional EMD methods}

As a nonlinear signal analysis method for processing nonlinear and non-stationary time series, 
291 EMD has been widely used in time series. To further verify the effectiveness of our EMD model, 292 we compare it with four widely used EMD models, namely EMD-ELM, EMD-SVR, EMD-SP-

293 SVR, and EMD-ANN. It is worth noting that in this study, these methods used the same way as 294 our proposed model, using EMD to decompose the wind speed, using a single classifier to predict 295 each IMF component separately, and adding all the prediction results to get the final prediction 296 wind speed. The prediction results and the error estimated results of these four EMD-based 297 methods and the proposed method are displayed in Fig. 5-6 and Table 6-9. Based on Fig. 5-6 and 298 Table 6-9, it can be observed that:

299 (1) Compared with the above-mentioned classic individual models, the performance of the EMD300 based method is significantly improved. Take wind station \#1 as an example, in the 1-step 301

302

303

304

305

306 forecasting, the value of RMSE of the EMD-based methods is around 0.60, while the classic individual model is around 1.20. After the wind speed is decomposed by EMD, the value of RMSE is reduced almost doubled.

(2) For wind station \#1, except for the MAE in the 3-step forecasting, the performance indicators obtained from the proposed model are significantly better than those EMD-based combined models. For the 3-step forecasting, the performance of EMD-SVR and EMD-SVR-SP in MAE is slightly better than the proposed combined model, but in other evaluation indicators, the proposed combined model achieves a significantly better performance. Furthermore, EMDANN is always worse in MAPE as compared with the other three combined models, with MAPE of $23.55 \%, 27.67 \%$, and $29.31 \%$ for 1 - to 3 -step forecasting.

(3) For wind station \#2, in 1- to 3-step wind speed forecasting, the proposed combined model obtains the best prediction results. The RMSE, MAE and MAPE in the 1-step forecasting are $0.5593,0.419$, and $17.10 \%$, respectively. In comparison, among the other four EMD-based combined models, the EMD-ELM and EMD-ANN models have similar prediction $21.83 \%, 25.3 \%, 27.86 \%$, respectively.

In total, the EMD-based method has obvious advantages over traditional methods, and the

\section{Discussion}

\section{Performance of SVR-SP and LassoCV on different IMFs}

321 According to the EMD principle, the frequency of the IMF components is from high to low. The 322 non-linear and non-stationary information of wind speed data is mainly concentrated in the high323 frequency IMF, and the low-frequency IMF presents a Sin-like function curve. Based on its 
324 characteristics, in this study we use SVR-SP and LassoCV to predict IMFs of different frequencies.

325 In order to verify the effectiveness of this hybrid EMD model, in this section, we take wind station

$326 \# 2$ as an example to analyze the performance of the two methods on different IMF components.

327 Table 10 lists the RMSE of SVR-SP and LassoCV on different IMF components. It is worth

328 mentioning that in multi-step prediction, the prediction accuracy of the first step is more important

329 than the other steps, which is of great significance for the accurate estimation of wind power. It

330 can be seen from Table 10 that SVR-SP can obtain significantly better performance than LassoCV

331 at high frequency (IMF1), while LassoCV can obtain better performance at low frequencies

332 (IMF2 IMF7, Trend), and its RMSE is already close to zero at IMF4. Moreover, SVR-SP has a

333 risk of overfitting when predicting low frequencies, resulting in poor performance. In total, the

334 proposed model that combines the EMD decomposition characteristics and the advantages of the

335 algorithm can achieve better performance than the traditional EMD model.

\section{Comparison of different signal decomposition techniques}

337 Besides EMD, Variational Mode Decomposition (VMD) and Ensemble Empirical Mode 338 Decomposition (EEMD) are also widely used in short-term wind speed forecasting. Here, we 339 analyze the impact of different signal decomposition techniques on the performance of our 340 proposed method. Table 11 shows the prediction performance of the three signal decomposition 341 techniques on two wind stations. For wind station \#1, it can be found that compared with VMD 342 and EEMD, EMD obtains the best RMSE value in the 1-step forecasting. The performance 343 obtained by VMD in the 1-step and 2-step forecasting is relatively close, but it drops significantly 344 in the 3-step forecasting. EEMD inherits from EMD, similar to EMD, as the step size increases, 345 the performance will decrease significantly. For wind station \#2, EMD also obtained the best 346 predictive performance. VMD has a similar conclusion on wind station \#1, and the performance 347 of the 1-step and 2-step forecasting is relatively close. It should be pointed out that in multi-step 348 forecasting, the 1-step forecasting is usually used for wind energy estimation, and other steps are 349 used to assist decision-making, so more attention is paid to the performance of the 1-step 350 forecasting.

\section{The impact of the number of selected features on performance}

352 Feature selection is used to remove redundant features in the study. However, the number of 353 selected significant features will more or less affect the short-term wind speed forecasting. In order 354 to ensure the stability in the complicated industrial system, we analyzed the performance of our 355 proposed method under the different number of selected features. Figure 7 shows the RMSE value 356 between the number of selected features and the performance of our proposed method. It should 
357 be pointed out that in the study based on the characteristics of EMD decomposition we use FS and

358 SVR to predict high-frequency component (i.e., $\mathrm{IMF}_{1}$ ), and use LassoCV to predict low-frequency

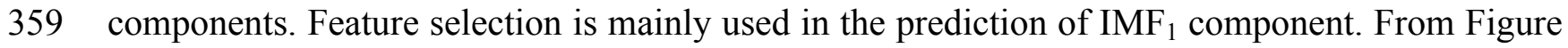

3607 , we can be seen that feature selection can slightly improve the performance of 1-step forecasting,

361 but has little effect on 1-step and 2-step forecasting. Overall, as the number of selected features

362 decreases, the generalization performance of the method will improve, but when the selected

363 features are too scarce, the performance will drop sharply due to the deletion of useful features. In

364 order to determine the appropriate number of features, by following (Bradley et al. 1998; Chizi et

365 al. 2009), this study uses cross-validation to select.

366

367

368

369

370

371

372

373

374

375

376

377

378

379

380

381

382

383

384

385

386

387

388

389

\section{Performance under different signal-to-noise ratios}

In the process of collecting wind speed, it is often affected by the environment and the anemometer itself, resulting in a certain amount of noise in the data. In order to verify the reliability of the method, we analyzed the prediction performance under different signal-to-noise ratios (SNRs). Figure 8 shows the 1-step to 3-step prediction performance of the method from 30 60db SNR. Take wind station \#1 as an example, it can be seen from Figure 8 that the performance of the proposed method is relatively stable under different signal-to-noise ratios. The RMSE value of 1step forecasting is about 0.6, the RMSE value of 2-step forecasting is about 0.75 , and the RMSE value of 3-step forecasting is about 0.85 . In general, as the signal-to-noise ratio increases, the prediction performance of the proposed method will be improved. Similar performance also exists on site \#2. These experimental results show that the proposed method can accurately predict wind speed under certain noise.

\section{Conclusions}

As a sustainable and renewable energy, wind power has attracted widespread attention and rapid development in recent years. Reliable and accurate wind speed forecasting will provide support for wind power planning and control. Due to the non-linearity and non-stationarity of wind, forecasting is still a difficult yet challenging problem. In the paper, we develop a new wind speed forecasting model based on EMD, FS, SVR and LassoCV. EMD is employed to extract IMFs from the original non-stationary wind speed time series. FS and SVR are combined to predict the highfrequency IMF. LassoCV is adopted to complete the prediction of low-frequency IMF and trend. By testing in two wind speeds obtained from Michigan, USA, the experimental results show that under 1- to 3-step forecasting the proposed model can achieve better prediction performance than the classic individual and traditional EMD combined models. Although the proposed model has achieved good performance, it still has some limitations. After the new data is updated, the model 
390

391

392

393

394

395

396

397

398

399

400

401

402

403

404

405

406

407

408

409

410

411

412

413

414

415

416

417

418

419

420

421

422

423

424

425

426

427

needs to be retrained. In future research, we will try to integrate online learning in our proposed method.

\section{References}

Ait Maatallah O, Achuthan A, Janoyan K, and Marzocca P. 2015. Recursive wind speed forecasting based on Hammerstein Auto-Regressive model. Applied Energy 145:191-197. https://doi.org/10.1016/j.apenergy.2015.02.032

Azeem A, Fatema N, Malik HJJoI, and Systems F. 2018. k-NN and ANN based deterministic and probabilistic wind speed forecasting intelligent approach. 35:5021-5031.

Bokde N, Feijóo A, Villanueva D, and Kulat KJE. 2019. A review on hybrid empirical mode decomposition models for wind speed and wind power prediction. 12:254.

Botha N, and Walt CMvd. 2017. Forecasting wind speed using support vector regression and feature selection. 2017 Pattern Recognition Association of South Africa and Robotics and Mechatronics (PRASA-RobMech). p 181-186.

Bradley PS, Mangasarian OL, and Street WN. 1998. Feature Selection via Mathematical Programming. INFORMS Journal on Computing 10:209-217.

Chandrashekar G, and Sahin F. 2014. A survey on feature selection methods. Computers \& Electrical Engineering 40:16-28. https://doi.org/10.1016/j.compeleceng.2013.11.024

Chen J, Zeng G-Q, Zhou W, Du W, Lu K-DJEC, and Management. 2018. Wind speed forecasting using nonlinear-learning ensemble of deep learning time series prediction and extremal optimization. 165:681-695.

Chizi B, Rokach L, and Maimon O. 2009. A survey of feature selection techniques. Encyclopedia of Data Warehousing and Mining, Second Edition: IGI Global, 1888-1895.

Drucker H, Burges CJ, Kaufman L, Smola AJ, and Vapnik V. 1997. Support vector regression machines. Advances in neural information processing systems. p 155-161.

Fu Y, Gao Z, Liu Y, Zhang A, and Yin X. 2020. Actuator and Sensor Fault Classification for Wind Turbine Systems Based on Fast Fourier Transform and Uncorrelated Multi-Linear Principal Component Analysis Techniques. Processes 8. 10.3390/pr8091066

Gao Z, and Liu X. 2021. An Overview on Fault Diagnosis, Prognosis and Resilient Control for Wind Turbine Systems. Processes 9. 10.3390/pr9020300

GLOBAL WECJB. 2020. Global Wind report 2019.

Heng J, Wang C, Zhao X, and Xiao LJS. 2016. Research and application based on adaptive boosting strategy and modified CGFPA algorithm: a case study for wind speed forecasting. 8:235.

Hong Y-Y, and Satriani TRA. 2020. Day-ahead spatiotemporal wind speed forecasting using robust design-based deep learning neural network. Energy 209:118441. https://doi.org/10.1016/j.energy.2020.118441

Hu J, and Wang J. 2015. Short-term wind speed prediction using empirical wavelet transform and $\begin{array}{llll}\text { Gaussian } & \text { process } & \text { 93:1456-1466. }\end{array}$ 
428

429

430

431

432

433

434

435

436

437

438

439

440

441

442

443

444

445

446

447

448

449

450

451

452

453

454

455

456

457

458

459

460

461

462

463

464

465

466

https://doi.org/10.1016/j.energy.2015.10.041

$\mathrm{Hu} \mathrm{Y}-\mathrm{L}$, Chen LJEc, and management. 2018. A nonlinear hybrid wind speed forecasting model using LSTM network, hysteretic ELM and Differential Evolution algorithm. 173:123142.

Huang NE, Shen Z, Long SR, Wu MC, Shih HH, Zheng Q, Yen N-C, Tung CC, Liu HHJPotRSoLSAm, physical, and sciences e. 1998. The empirical mode decomposition and the Hilbert spectrum for nonlinear and non-stationary time series analysis. 454:903-995.

Hung JC, Yen NY, and Li K-C. 2016. Frontier Computing: Theory, Technologies and Applications: Springer.

Kavasseri RG, and Seetharaman K. 2009. Day-ahead wind speed forecasting using f-ARIMA models. Renewable Energy 34:1388-1393. https://doi.org/10.1016/j.renene.2008.09.006

Khodayar M, and Wang J. 2019. Spatio-Temporal Graph Deep Neural Network for Short-Term Wind Speed Forecasting. IEEE Transactions on Sustainable Energy 10:670-681. 10.1109/TSTE.2018.2844102

Khodayar M, Wang J, and Manthouri M. 2019. Interval Deep Generative Neural Network for Wind Speed Forecasting. IEEE Transactions on Smart Grid 10:3974-3989.

Khosravi A, Koury R, Machado L, Pabon JJSET, and Assessments. 2018. Prediction of wind speed and wind direction using artificial neural network, support vector regression and adaptive neuro-fuzzy inference system. 25:146-160.

Kong X, Liu X, Shi R, and Lee KY. 2015. Wind speed prediction using reduced support vector machines with feature selection. Neurocomputing 169:449-456. https://doi.org/10.1016/i.neucom.2014.09.090

Li C, Xiao Z, Xia X, Zou W, and Zhang CJAE. 2018a. A hybrid model based on synchronous optimisation for multi-step short-term wind speed forecasting. 215:131-144.

Li H, Wang J, Lu H, and Guo ZJRE. 2018b. Research and application of a combined model based on variable weight for short term wind speed forecasting. 116:669-684.

Liu H, Mi X, Li Y, Duan Z, and Xu YJRe. 2019a. Smart wind speed deep learning based multistep forecasting model using singular spectrum analysis, convolutional Gated Recurrent Unit network and Support Vector Regression. 143:842-854.

Liu H, Mi X, Li YJEC, and Management. 2018a. Smart deep learning based wind speed prediction model using wavelet packet decomposition, convolutional neural network and convolutional long short term memory network. 166:120-131.

Liu H, Mi X, Li YJEC, and Management. 2018b. Smart multi-step deep learning model for wind speed forecasting based on variational mode decomposition, singular spectrum analysis, LSTM network and ELM. 159:54-64.

Liu M, Cao Z, Zhang J, Wang L, Huang C, and Luo X. 2020. Short-term wind speed forecasting based on the Jaya-SVM model. International Journal of Electrical Power \& Energy Systems 121:106056. https://doi.org/10.1016/j.ijepes.2020.106056

Liu Y, Shi H, Huang S, Chen X, Zhou H, Chang H, Xia Y, Wang G, Yang XJQiim, and surgery.

Peer] Comput. Sci. reviewing PDF | (CS-2021:02:57863:2:0:NEW 10 Aug 2021) 
467

468

469

470

471

472

473

474

475

476

477

478

479

480

481

482

483

484

485

486

487

488

489

490

491

492

493

494

495

496

497

498

499

500

501

502

503

504

505

$2019 b$. Early prediction of acute xerostomia during radiation therapy for nasopharyngeal cancer based on delta radiomics from CT images. 9:1288.

Lydia M, Kumar SS, Selvakumar AI, Kumar GEPJEC, and Management. 2016a. Linear and nonlinear autoregressive models for short-term wind speed forecasting. 112:115-124.

Lydia M, Suresh Kumar S, Immanuel Selvakumar A, and Edwin Prem Kumar G. 2016b. Linear and non-linear autoregressive models for short-term wind speed forecasting. Energy Conversion and Management

112:115-124. https://doi.org/10.1016/j.enconman.2016.01.007

Nair KR, Vanitha V, and Jisma M. 2017. Forecasting of wind speed using ann, arima and hybrid models. 2017 International Conference on Intelligent Computing, Instrumentation and Control Technologies (ICICICT): IEEE. p 170-175.

Niu T, Wang J, Zhang K, and Du P. 2018. Multi-step-ahead wind speed forecasting based on optimal feature selection and a modified bat algorithm with the cognition strategy. Renewable Energy 118:213-229. https://doi.org/10.1016/j.renene.2017.10.075

Paramasivan SK, and Lopez DJIJoRER. 2016. Forecasting of wind speed using feature selection and neural networks. 6:833-837.

Park T, and Casella GJJotASA. 2008. The bayesian lasso. 103:681-686.

Poggi P, Muselli M, Notton G, Cristofari C, and Louche A. 2003. Forecasting and simulating wind speed in Corsica by using an autoregressive model. Energy Conversion and Management 44:3177-3196. https://doi.org/10.1016/S0196-8904(03)00108-0

Santamaría-Bonfil G, Reyes-Ballesteros A, and Gershenson CJRE. 2016. Wind speed forecasting for wind farms: A method based on support vector regression. 85:790-809.

Sun W, and Wang Y. 2018. Short-term wind speed forecasting based on fast ensemble empirical mode decomposition, phase space reconstruction, sample entropy and improved backpropagation neural network. Energy Conversion and Management 157:1-12. https://doi.org/10.1016/j.enconman.2017.11.067

Tascikaraoglu A, Sanandaji BM, Poolla K, and Varaiya P. 2016. Exploiting sparsity of interconnections in spatio-temporal wind speed forecasting using Wavelet Transform. Applied Energy 165:735-747. https://doi.org/10.1016/j.apenergy.2015.12.082

Tibshirani RJJotRSSSB. 1996. Regression shrinkage and selection via the lasso. 58:267-288.

Torres JL, Garcia A, De Blas M, and De Francisco AJSe. 2005. Forecast of hourly average wind speed with ARMA models in Navarre (Spain). 79:65-77.

Wang HZ, Wang GB, Li GQ, Peng JC, and Liu YT. 2016a. Deep belief network based deterministic and probabilistic wind speed forecasting approach. Applied Energy 182:8093. https://doi.org/10.1016/j.apenergy.2016.08.108

Wang J, and Hu JJE. 2015. A robust combination approach for short-term wind speed forecasting and analysis-Combination of the ARIMA (Autoregressive Integrated Moving Average), ELM (Extreme Learning Machine), SVM (Support Vector Machine) and LSSVM (Least Square SVM) forecasts using a GPR (Gaussian Process Regression) model. 93:41-56. 
506 Wang L, Li X, and Bai Y. 2018. Short-term wind speed prediction using an extreme learning 507 machine model with error correction. Energy Conversion and Management 162:239-250. $508 \quad$ https://doi.org/10.1016/j.enconman.2018.02.015

509 Wang S, Zhang N, Wu L, and Wang Y. 2016b. Wind speed forecasting based on the hybrid 510 ensemble empirical mode decomposition and GA-BP neural network method. Renewable 511 Energy 94:629-636. https://doi.org/10.1016/j.renene.2016.03.103

512 Wang X. 2017. Forecasting short-term wind speed using support vector machine with particle 513 swarm optimization. 2017 International Conference on Sensing, Diagnostics, Prognostics, 514 and Control (SDPC): IEEE. p 241-245.

515 Yu C, Li Y, and Zhang M. 2017. An improved Wavelet Transform using Singular Spectrum $516 \quad$ Analysis for wind speed forecasting based on Elman Neural Network. Energy Conversion 517 and Management 148:895-904. https://doi.org/10.1016/j.enconman.2017.05.063

518 Zhang D, Peng X, Pan K, and Liu Y. 2019. A novel wind speed forecasting based on hybrid 519 decomposition and online sequential outlier robust extreme learning machine. Energy Conversion and Management 180:338-357. https://doi.org/10.1016/j.enconman.2018.10.089

Ziqiang Z, and Puthusserypady S. 2007. Analysis of schizophrenic EEG synchrony using empirical mode decomposition. 2007 15th International Conference on Digital Signal Processing: IEEE. p 131-134.

525

526 
Figure 1

The whole process of the proposed model. 
a.

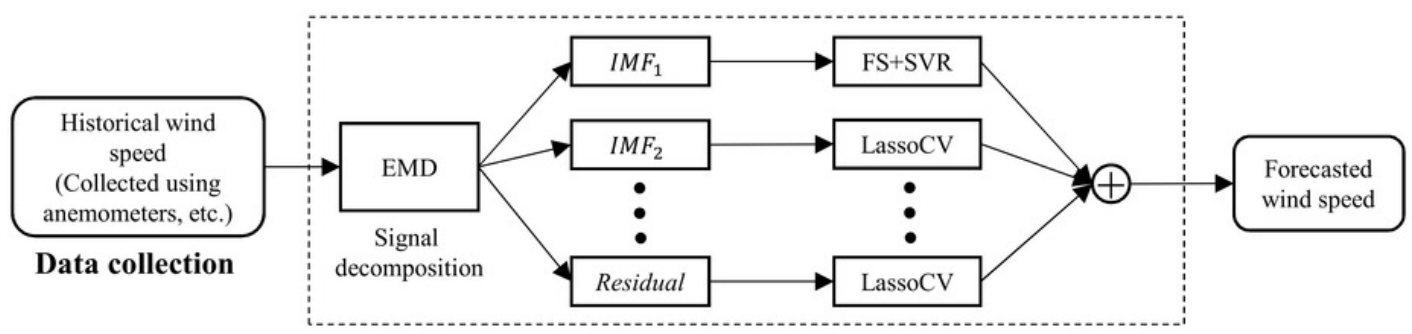

Short-term wind speed forecasting model

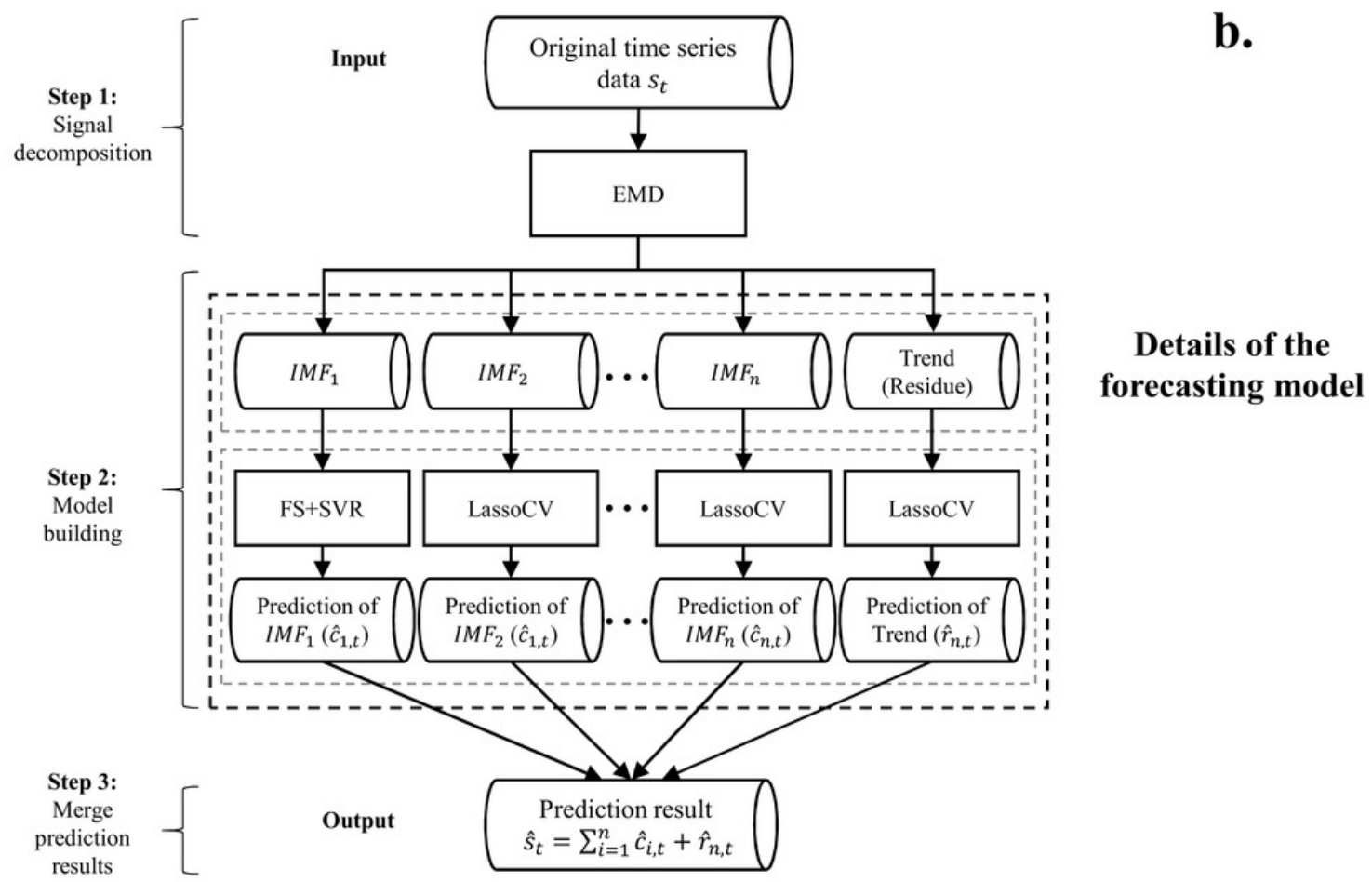

c.

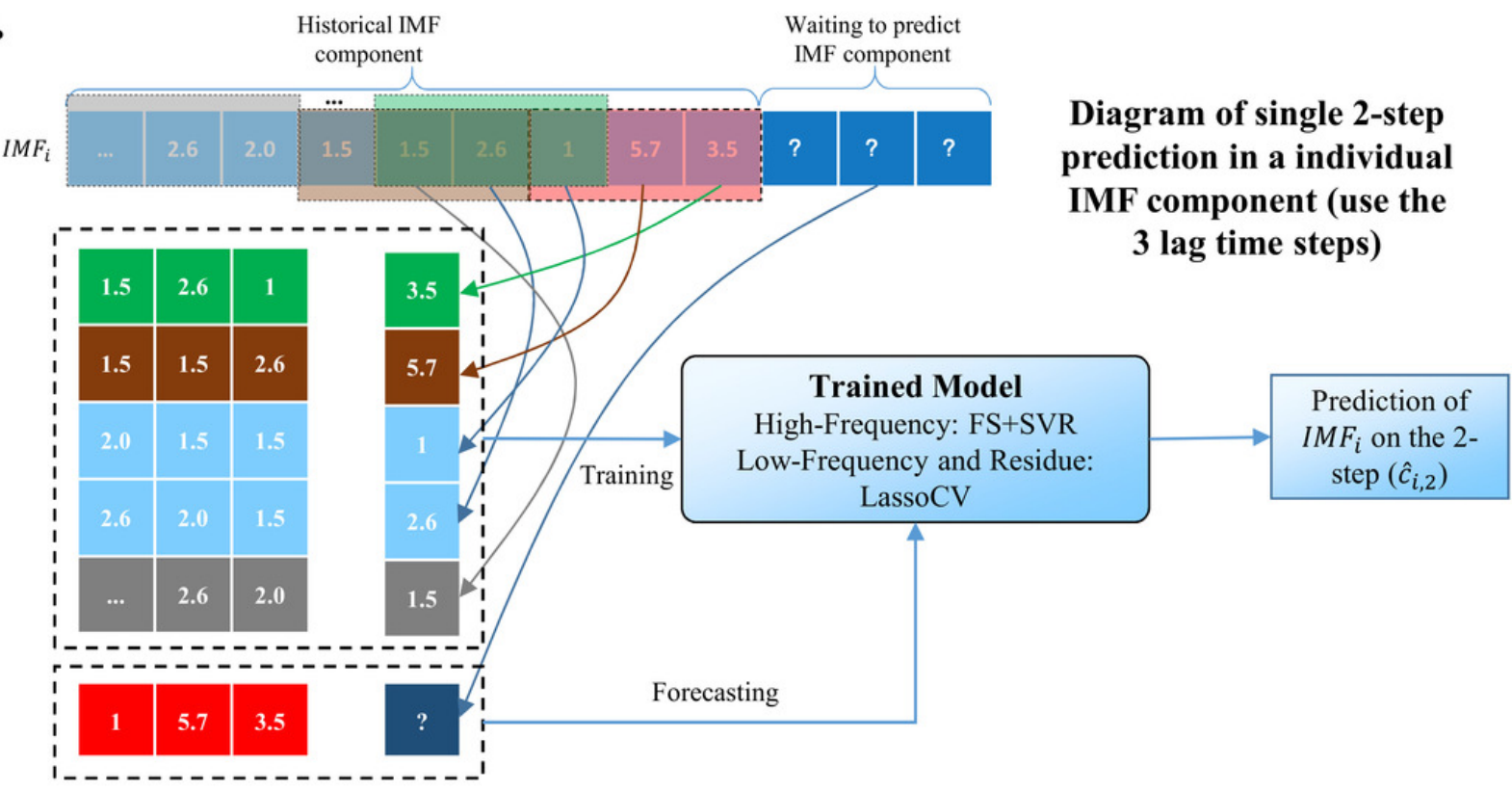


Figure 2

Wind speed collected from wind stations \#1 and \#2.

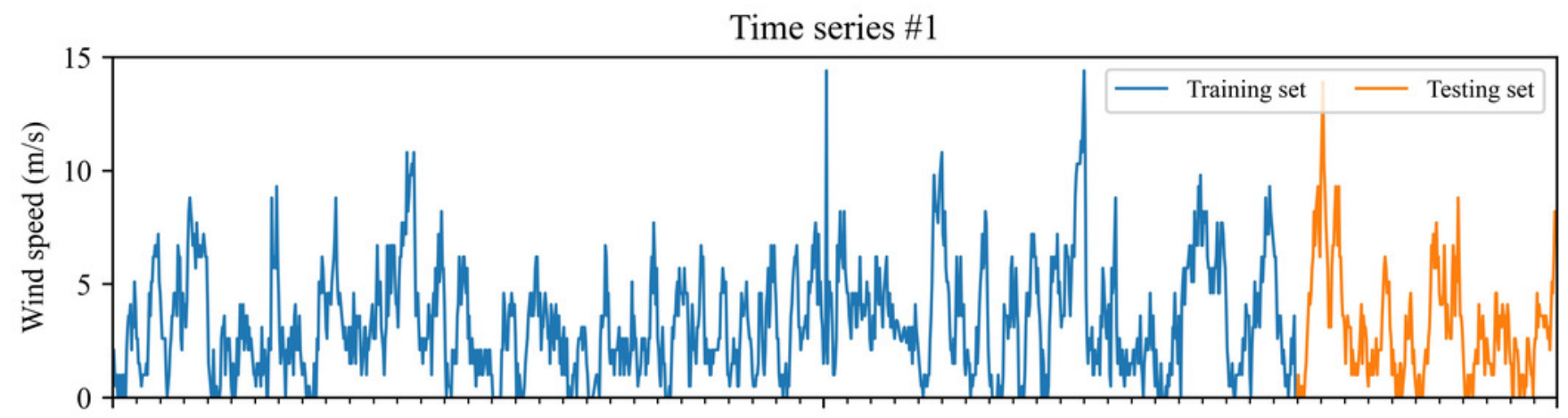

Time series \#2

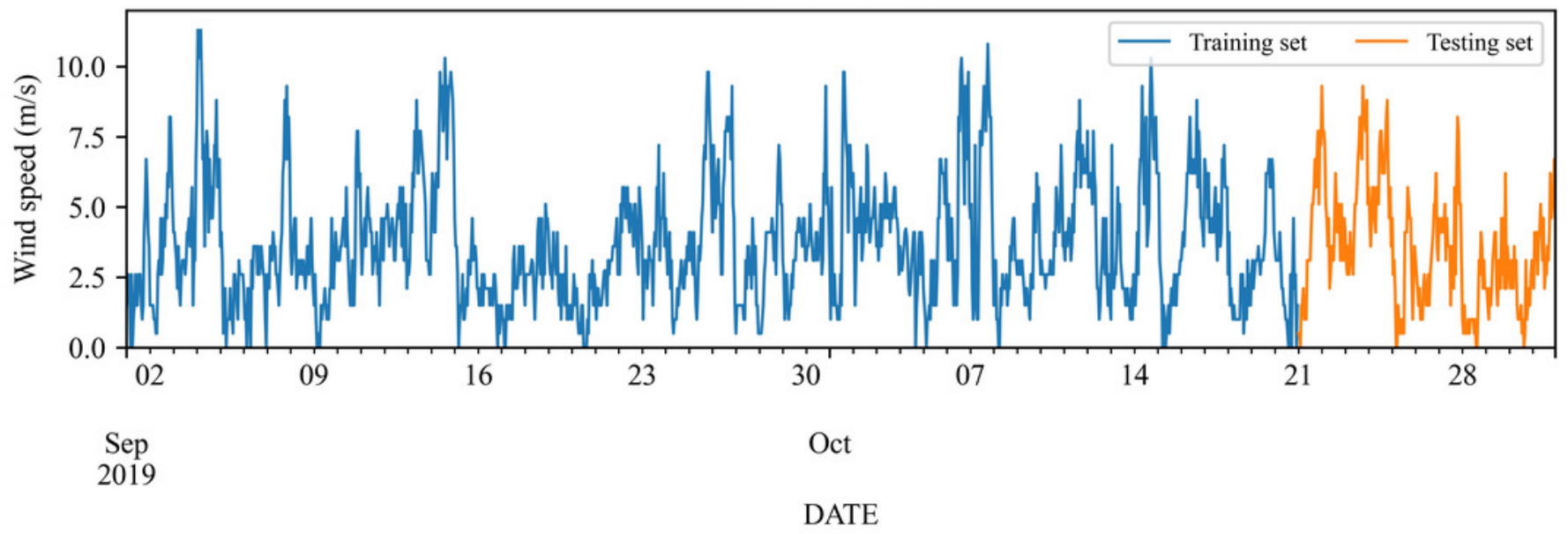


Figure 3

The prediction of the classic individual models at wind station \#1.
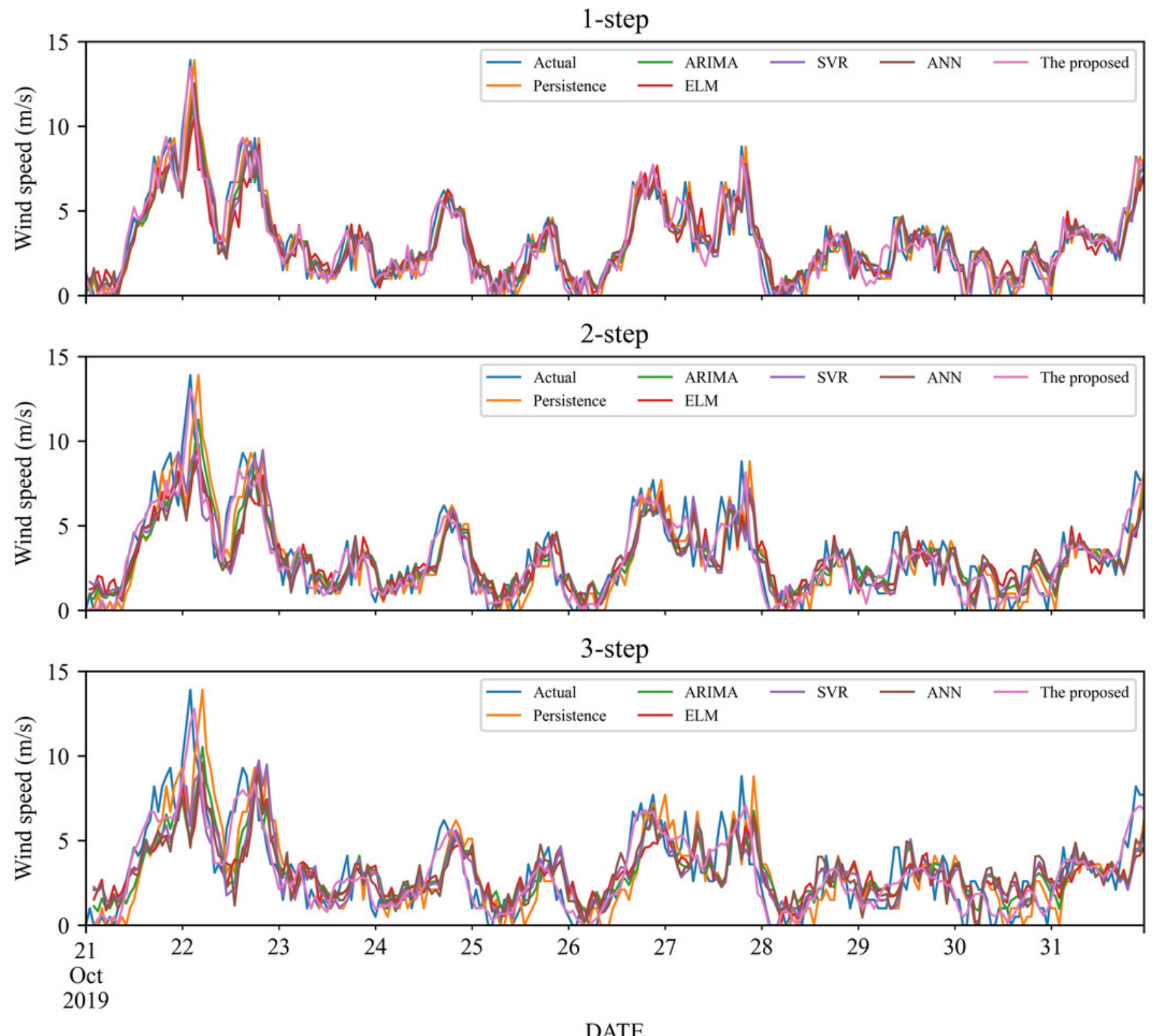
Figure 4

The prediction of the classic individual models at wind station \#2.
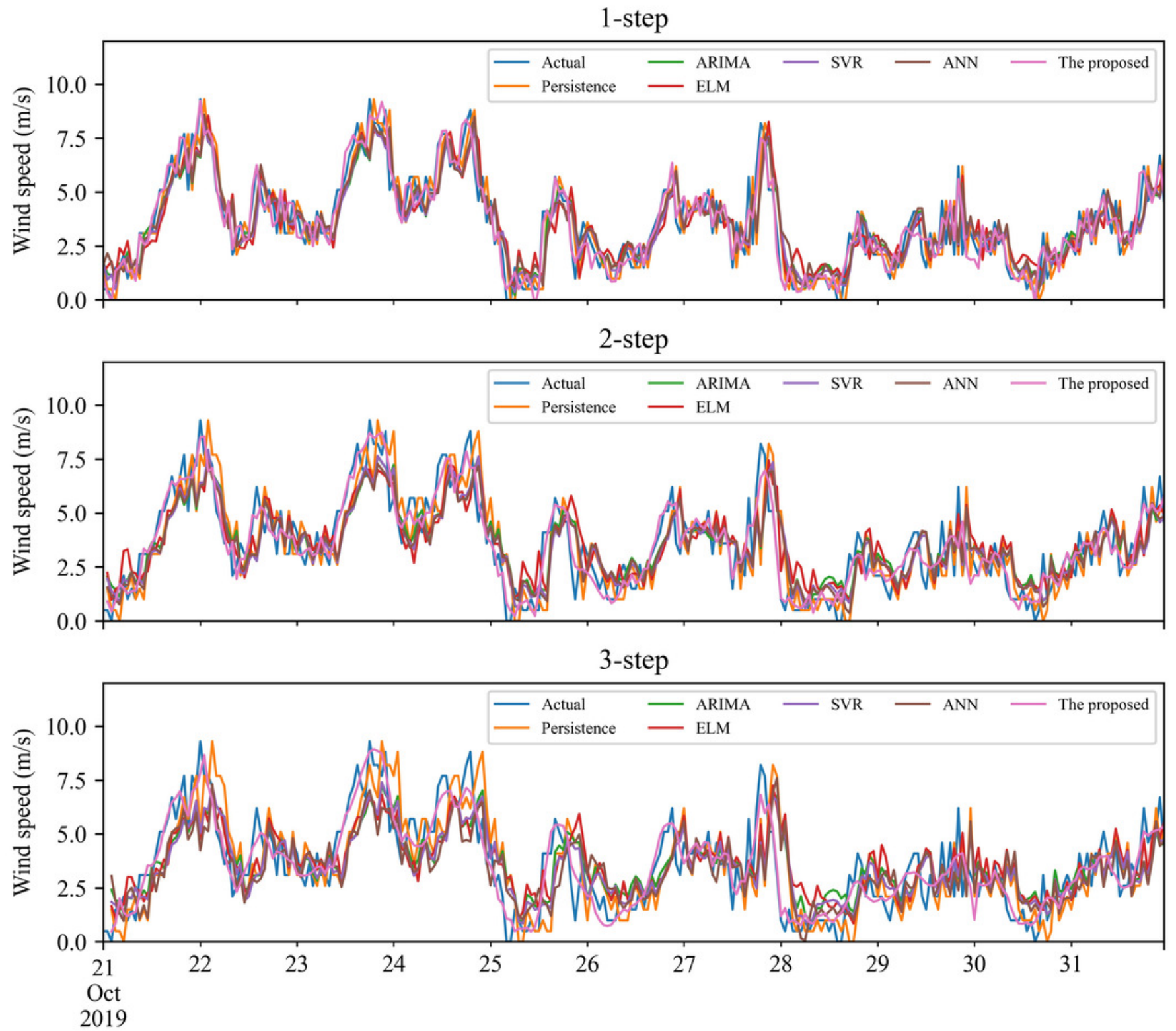

DATE 
Figure 5

The prediction of different combination models at wind station \#1.
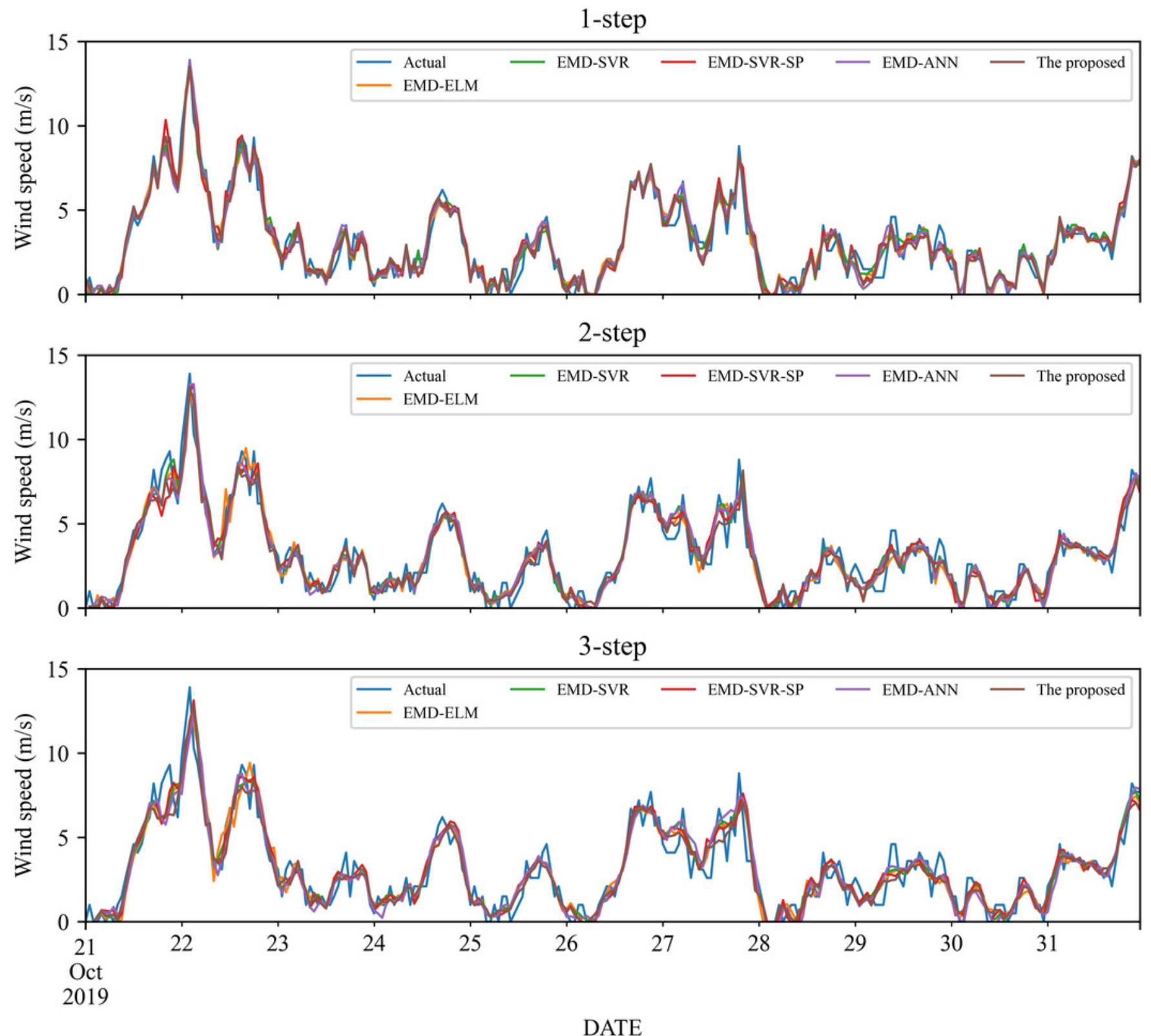
Figure 6

The prediction of different combination models at wind station \#2.
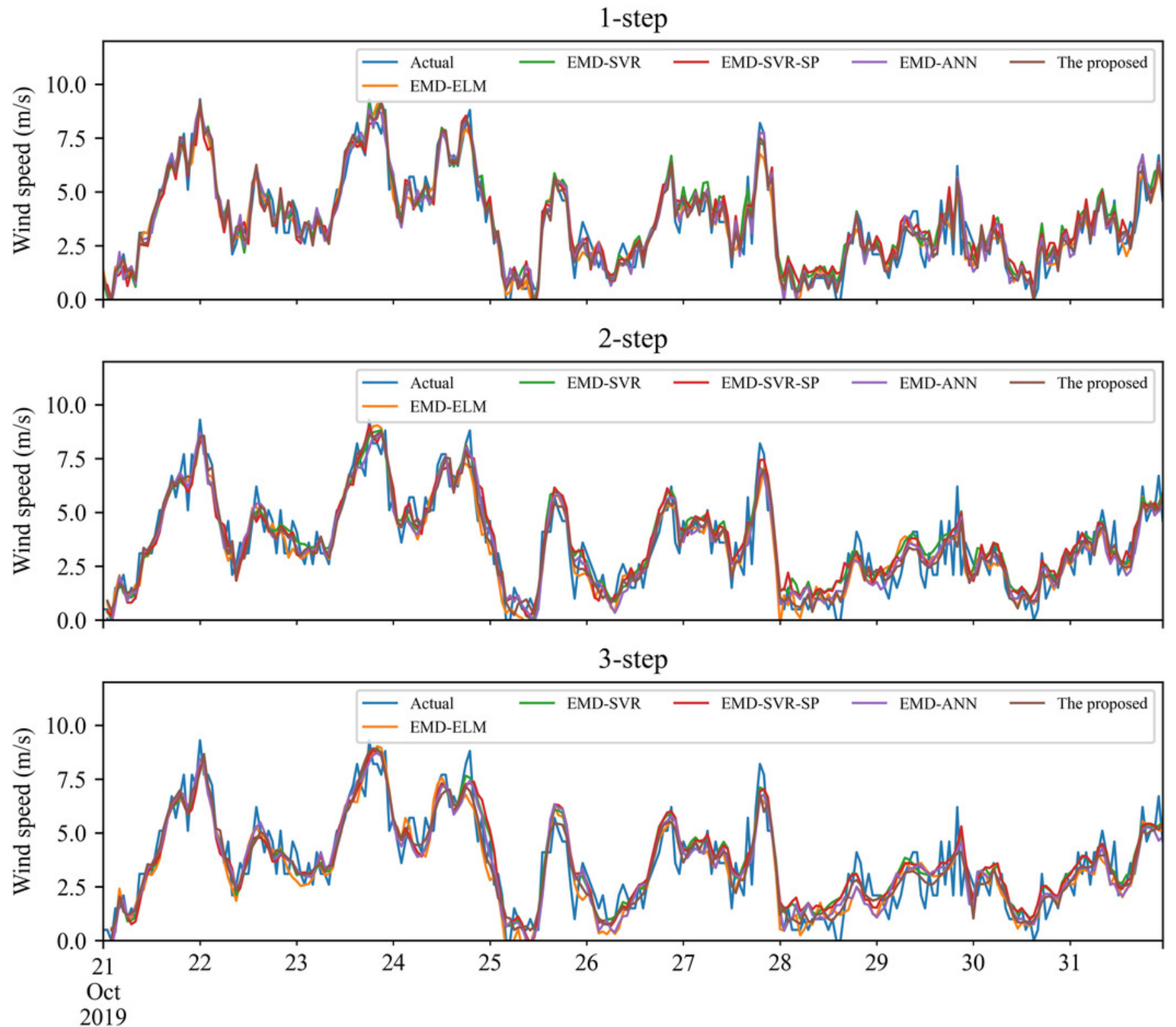

DATE 
Figure 7

The RMSE between the number of selected features and the performance of the proposed method.

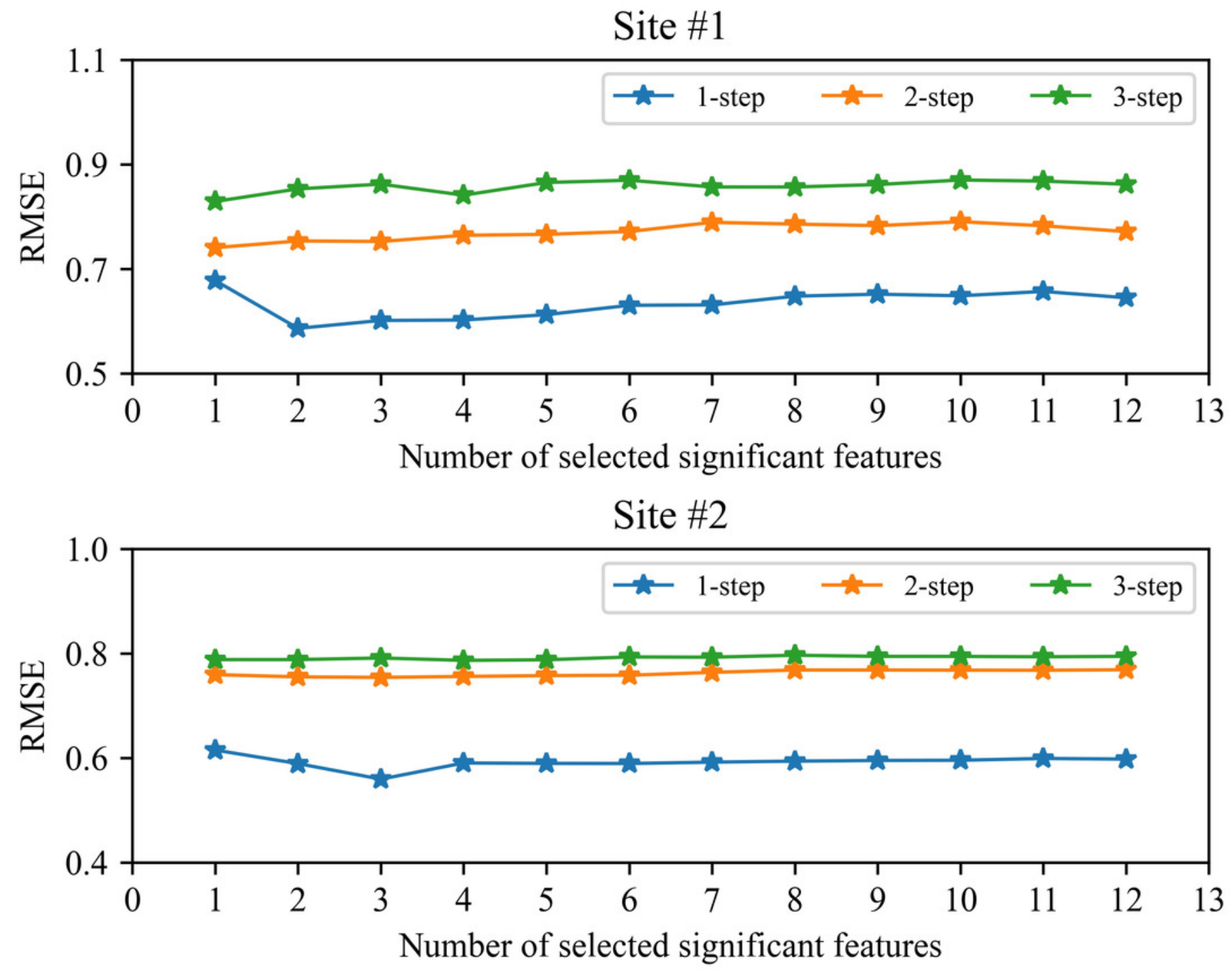


Figure 8

The RMSE of the proposed method at 30 60db SNR.
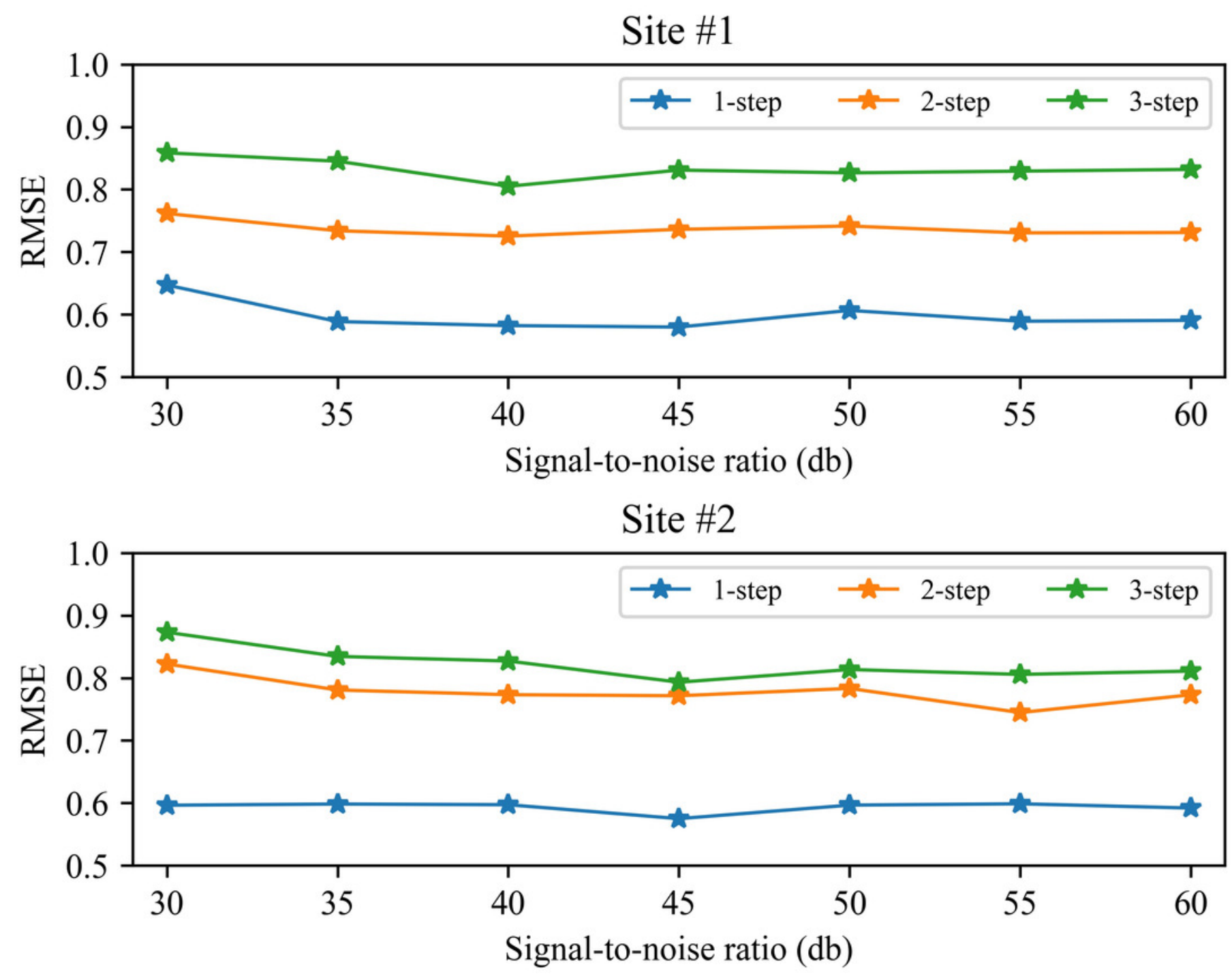


\section{Table $\mathbf{1}$ (on next page)}

Wind speed statistics at wind stations \#1 and \#2. 
1

\begin{tabular}{|c|c|c|c|c|c|c|c|c|}
\hline \multirow{2}{*}{$\begin{array}{l}\text { Wind } \\
\text { station }\end{array}$} & \multirow{2}{*}{ Dataset } & \multirow{2}{*}{ Date } & \multicolumn{6}{|c|}{ Statistical indicators } \\
\hline & & & $\operatorname{Mean}(\mathrm{m} / \mathrm{s})$ & $\operatorname{Max}(m / s)$ & $\operatorname{Min}(\mathbf{m} / \mathbf{s})$ & Std. & Stew & Kurt. \\
\hline \multirow{2}{*}{ Site \#1 } & $\begin{array}{c}\text { Training } \\
\text { set }\end{array}$ & $\begin{array}{c}\text { Sept. 1, 2019 } \\
\text { Oct. 20, } 2019(\sim 83 \%)\end{array}$ & 3.2975 & 14.4 & 0 & 2.378 & 0.871 & 0.865 \\
\hline & Testing set & $\begin{array}{c}\text { Oct. } 21,2019 \sim \\
\text { Oct. } 31,2019(\sim 17 \%)\end{array}$ & 3.1614 & 13.9 & 0 & 2.486 & 1.108 & 1.312 \\
\hline \multirow{2}{*}{ Site \#2 } & $\begin{array}{c}\text { Training } \\
\text { set }\end{array}$ & $\begin{array}{c}\text { Sept. 1, } 2019 \sim \\
\text { Oct. } 20,2020(\sim 83 \%)\end{array}$ & 3.6919 & 11.3 & 0 & 2.183 & 0.807 & 0.353 \\
\hline & Testing set & $\begin{array}{c}\text { Oct. } 21,2019 \sim \\
\text { Oct. } 31,2020(\sim 17 \%)\end{array}$ & 3.5667 & 9.3 & 0 & 2.118 & 0.500 & -0.318 \\
\hline
\end{tabular}

2 


\section{Table 2 (on next page)}

The error result of the classic individual models at wind station \#1. 


\begin{tabular}{|c|c|c|c|c|c|c|c|c|c|}
\hline \multirow{2}{*}{ Models } & \multicolumn{3}{|c|}{ 1-step } & \multicolumn{3}{|c|}{ 2-step } & \multicolumn{3}{|c|}{ 3-step } \\
\hline & RMSE & MAE & MAPE (\%) & RMSE & MAE & MAPE (\%) & RMSE & MAE & MAPE (\%) \\
\hline Persistence & 1.1892 & 0.8996 & 36.20 & 1.5892 & 1.2221 & 49.65 & 1.9008 & 1.4687 & 57.64 \\
\hline ARIMA & 1.1724 & 0.9010 & 34.25 & 1.5182 & 1.1561 & 45.25 & 1.7647 & 1.3569 & 53.79 \\
\hline ELM & 1.2705 & 0.9724 & 36.20 & 1.5500 & 1.1729 & 46.55 & 1.8109 & 1.3603 & 55.18 \\
\hline SVR & 1.1739 & 0.9024 & 34.87 & 1.5676 & 1.1928 & 46.71 & 1.7832 & 1.3376 & 52.78 \\
\hline ANN & 1.1984 & 0.9354 & 36.24 & 1.5338 & 1.1615 & 45.79 & 1.8427 & 1.3906 & 55.70 \\
\hline The proposed & 0.5859 & 0.4426 & 21.11 & 0.7531 & 0.5848 & 24.78 & 0.8528 & 0.6798 & 27.55 \\
\hline
\end{tabular}




\section{Table 3 (on next page)}

The error result of the classic individual models at wind station \#2. 


\begin{tabular}{|c|c|c|c|c|c|c|c|c|c|}
\hline \multirow{2}{*}{ Models } & \multicolumn{3}{|c|}{ 1-step } & \multicolumn{3}{|c|}{ 2-step } & \multicolumn{3}{|c|}{ 3-step } \\
\hline & RMSE & MAE & MAPE (\%) & RMSE & MAE & MAPE (\%) & RMSE & MAE & MAPE (\%) \\
\hline Persistence & 1.2720 & 0.9739 & 35.98 & 1.4292 & 1.0947 & 41.02 & 1.6700 & 1.3073 & 47.99 \\
\hline ARIMA & 1.1609 & 0.9302 & 38.71 & 1.3214 & 1.0430 & 45.43 & 1.5257 & 1.2188 & 53.05 \\
\hline ELM & 1.2528 & 1.0188 & 44.81 & 1.3657 & 1.0915 & 51.40 & 1.5867 & 1.2849 & 60.12 \\
\hline SVR & 1.1602 & 0.9218 & 36.51 & 1.3115 & 1.0360 & 43.63 & 1.5018 & 1.2008 & 49.91 \\
\hline ANN & 1.1901 & 0.9460 & 40.62 & 1.3116 & 1.0330 & 43.72 & 1.6345 & 1.2798 & 52.18 \\
\hline The proposed & 0.5593 & 0.4193 & 17.10 & 0.7540 & 0.5966 & 22.99 & 0.7911 & 0.6437 & 24.59 \\
\hline
\end{tabular}




\section{Table 4(on next page)}

The improvement rate of the proposed model relative to the classic individual models at wind station \#1. 


\begin{tabular}{llccc}
\hline \hline Models & & 1-step & 2-step & 3-step \\
\hline Persistence & $\mathrm{P}_{\text {RMSE }}(\%)$ & 102.98 & 111.04 & 122.89 \\
& $\mathrm{P}_{\text {MAE }}(\%)$ & 103.24 & 108.97 & 116.05 \\
& $\mathrm{P}_{\text {MAPE }}(\%)$ & 71.47 & 100.34 & 109.26 \\
& & & & \\
ARIMA & $\mathrm{P}_{\text {RMSE }}(\%)$ & 100.11 & 101.60 & 106.94 \\
& $\mathrm{P}_{\text {MAE }}(\%)$ & 103.55 & 97.69 & 99.60 \\
& $\mathrm{P}_{\text {MAPE }}(\%)$ & 62.20 & 82.58 & 95.26 \\
& & & & \\
ELM & $\mathrm{P}_{\text {RMSE }}(\%)$ & 116.85 & 105.83 & 112.35 \\
& $\mathrm{P}_{\text {MAE }}(\%)$ & 119.68 & 100.57 & 100.11 \\
& $\mathrm{P}_{\text {MAPE }}(\%)$ & 71.44 & 87.82 & 100.31 \\
& & & & \\
SVR & $\mathrm{P}_{\text {RMSE }}(\%)$ & 100.36 & 108.16 & 109.11 \\
& $\mathrm{P}_{\text {MAE }}(\%)$ & 103.87 & 103.96 & 96.76 \\
& $\mathrm{P}_{\text {MAPE }}(\%)$ & 65.15 & 88.48 & 91.62 \\
& & & & \\
ANN & $\mathrm{P}_{\text {RMSE }}(\%)$ & 104.54 & 103.68 & 116.09 \\
& $\mathrm{P}_{\text {MAE }}(\%)$ & 111.33 & 98.62 & 104.56 \\
& $\mathrm{P}_{\text {MAPE }}(\%)$ & 71.63 & 84.78 & 102.23 \\
\hline \hline
\end{tabular}




\section{Table 5 (on next page)}

The improvement rate of the proposed model relative to the classic individual models at wind station \#2. 


\begin{tabular}{llccc}
\hline \hline Models & & 1-step & 2-step & 3-step \\
\hline Persistence & $\mathrm{P}_{\text {RMSE }}(\%)$ & 127.42 & 89.54 & 111.11 \\
& $\mathrm{P}_{\text {MAE }}(\%)$ & 132.24 & 83.49 & 103.08 \\
& $\mathrm{P}_{\text {MAPE }}(\%)$ & 110.33 & 78.38 & 95.19 \\
& & & & \\
ARIMA & $\mathrm{P}_{\text {RMSE }}(\%)$ & 107.55 & 75.25 & 92.86 \\
& $\mathrm{P}_{\text {MAE }}(\%)$ & 121.83 & 74.83 & 89.35 \\
& $\mathrm{P}_{\text {MAPE }}(\%)$ & 126.31 & 97.56 & 115.77 \\
& & & & \\
ELM & $\mathrm{P}_{\text {RMSE }}(\%)$ & 123.99 & 81.12 & 100.59 \\
& $\mathrm{P}_{\text {MAE }}(\%)$ & 142.95 & 82.96 & 99.60 \\
& $\mathrm{P}_{\text {MAPE }}(\%)$ & 161.98 & 123.54 & 144.54 \\
& & & & \\
SVR & $\mathrm{P}_{\text {RMSE }}(\%)$ & 107.43 & 73.93 & 89.84 \\
& $\mathrm{P}_{\text {MAE }}(\%)$ & 119.81 & 73.66 & 86.54 \\
& $\mathrm{P}_{\text {MAPE }}(\%)$ & 113.43 & 89.76 & 103.00 \\
& & & & \\
ANN & $\mathrm{P}_{\text {RMSE }}(\%)$ & 112.78 & 73.95 & 106.62 \\
& $\mathrm{P}_{\text {MAE }}(\%)$ & 125.58 & 73.16 & 98.82 \\
& $\mathrm{P}_{\text {MAPE }}(\%)$ & 137.50 & 90.12 & 112.23 \\
\hline \hline
\end{tabular}




\section{Table 6(on next page)}

The error result of different combination models at wind station \#1. 


\begin{tabular}{|c|c|c|c|c|c|c|c|c|c|}
\hline \multirow{2}{*}{ Models } & \multicolumn{3}{|c|}{ 1-step } & \multicolumn{3}{|c|}{ 2-step } & \multicolumn{3}{|c|}{ 3-step } \\
\hline & RMSE & MAE & MAPE (\%) & RMSE & MAE & MAPE (\%) & RMSE & MAE & MAPE (\%) \\
\hline EMD-ELM & 0.6400 & 0.5128 & 22.63 & 0.7854 & 0.6316 & 27.22 & 0.8746 & 0.6937 & 29.02 \\
\hline EMD-SVR & 0.6379 & 0.5120 & 23.32 & 0.7768 & 0.6181 & 27.09 & 0.8583 & 0.6749 & 28.48 \\
\hline EMD-SVR-SP & 0.6310 & 0.4867 & 23.03 & 0.7987 & 0.6141 & 26.30 & 0.8591 & 0.6762 & 28.66 \\
\hline EMD-ANN & 0.6342 & 0.5055 & 23.55 & 0.7879 & 0.6221 & 27.67 & 0.8987 & 0.7040 & 29.31 \\
\hline The proposed & 0.5859 & 0.4426 & 21.11 & 0.7531 & 0.5848 & 24.78 & 0.8528 & 0.6798 & 27.55 \\
\hline
\end{tabular}




\section{Table 7 (on next page)}

The error result of different combination models at wind station \#2. 


\begin{tabular}{|c|c|c|c|c|c|c|c|c|c|}
\hline \multirow{2}{*}{ Models } & \multicolumn{3}{|c|}{ 1-step } & \multicolumn{3}{|c|}{ 2-step } & \multicolumn{3}{|c|}{ 3-step } \\
\hline & RMSE & MAE & MAPE (\%) & RMSE & MAE & MAPE (\%) & RMSE & MAE & MAPE (\%) \\
\hline EMD-ELM & 0.6560 & 0.5283 & 21.59 & 0.8199 & 0.6669 & 27.49 & 0.8775 & 0.7096 & 27.65 \\
\hline EMD-SVR & 0.6567 & 0.5233 & 24.88 & 0.8317 & 0.6736 & 29.85 & 0.8508 & 0.6986 & 30.52 \\
\hline EMD-SVR-SP & 0.6437 & 0.4972 & 24.06 & 0.8211 & 0.6718 & 28.53 & 0.8894 & 0.7264 & 32.31 \\
\hline EMD-ANN & 0.6397 & 0.5046 & 21.83 & 0.7927 & 0.6373 & 25.34 & 0.8520 & 0.6934 & 27.86 \\
\hline The proposed & 0.5593 & 0.4193 & 17.10 & 0.7540 & 0.5966 & 22.99 & 0.7911 & 0.6437 & 24.59 \\
\hline
\end{tabular}




\section{Table 8 (on next page)}

The improvement rate of the proposed model relative to other combined models at wind station \#1. 


\begin{tabular}{llccc}
\hline \hline Models & & 1-step & 2-step & 3-step \\
\hline EMD-ELM & $\mathrm{P}_{\mathrm{RMSE}}(\%)$ & 9.23 & 4.30 & 2.56 \\
& $\mathrm{P}_{\mathrm{MAE}}(\%)$ & 15.85 & 8.00 & 2.05 \\
& $\mathrm{P}_{\mathrm{MAPE}}(\%)$ & 7.20 & 9.82 & 5.36 \\
& & & & \\
EMD-SVR & $\mathrm{P}_{\mathrm{RMSE}}(\%)$ & 8.88 & 3.16 & 0.65 \\
& $\mathrm{P}_{\mathrm{MAE}}(\%)$ & 15.67 & 5.69 & -0.72 \\
& $\mathrm{P}_{\mathrm{MAPE}}(\%)$ & 10.43 & 9.31 & 3.41 \\
& & & & \\
EMD-SVR-SP & $\mathrm{P}_{\mathrm{RMSE}}(\%)$ & 7.70 & 6.06 & 0.74 \\
& $\mathrm{P}_{\mathrm{MAE}}(\%)$ & 9.95 & 5.01 & -0.53 \\
& $\mathrm{P}_{\mathrm{MAPE}}(\%)$ & 9.09 & 6.10 & 4.05 \\
& & & & \\
EMD-ANN & $\mathrm{P}_{\mathrm{RMSE}}(\%)$ & 8.25 & 4.63 & 5.39 \\
& $\mathrm{P}_{\mathrm{MAE}}(\%)$ & 14.21 & 6.38 & 3.56 \\
& $\mathrm{P}_{\mathrm{MAPE}}(\%)$ & 11.52 & 11.67 & 6.40 \\
\hline \hline
\end{tabular}

1 


\section{Table 9 (on next page)}

The improvement rate of the proposed model relative to other combined models at wind station \#2. 


\begin{tabular}{|c|c|c|c|c|}
\hline Models & & 1-step & 2-step & 3-step \\
\hline \multirow[t]{3}{*}{ EMD-ELM } & $\mathrm{P}_{\mathrm{RMSE}}(\%)$ & 17.29 & 8.74 & 10.93 \\
\hline & $\mathrm{P}_{\mathrm{MAE}}(\%)$ & 25.98 & 11.78 & 10.23 \\
\hline & $\mathrm{P}_{\mathrm{MAPE}}(\%)$ & 26.20 & 19.55 & 12.46 \\
\hline \multirow[t]{3}{*}{ EMD-SVR } & $\mathrm{P}_{\mathrm{RMSE}}(\%)$ & 17.41 & 10.30 & 7.56 \\
\hline & $\mathrm{P}_{\mathrm{MAE}}(\%)$ & 24.80 & 12.91 & 8.52 \\
\hline & $\mathrm{P}_{\mathrm{MAPE}}(\%)$ & 45.48 & 29.81 & 24.15 \\
\hline \multirow[t]{3}{*}{ EMD-SVR-SP } & $\mathrm{P}_{\mathrm{RMSE}}(\%)$ & 15.09 & 8.90 & 12.43 \\
\hline & $\mathrm{P}_{\mathrm{MAE}}(\%)$ & 18.58 & 12.61 & 12.84 \\
\hline & $\mathrm{P}_{\mathrm{MAPE}}(\%)$ & 40.64 & 24.08 & 31.42 \\
\hline \multirow[t]{3}{*}{ EMD-ANN } & $\mathrm{P}_{\mathrm{RMSE}}(\%)$ & 14.37 & 5.12 & 7.71 \\
\hline & $\mathrm{P}_{\mathrm{MAE}}(\%)$ & 20.33 & 6.83 & 7.72 \\
\hline & $\mathrm{P}_{\text {MAPE }}(\%)$ & 27.62 & 10.22 & 13.29 \\
\hline
\end{tabular}




\section{Table $\mathbf{1 0}$ (on next page)}

The RMSE of SVR-SP and LassoCV on different IMF components at wind station \#2. 


\begin{tabular}{cccccccccc}
\hline \hline Steps & Models & IMF1 & IMF2 & IMF3 & IMF4 & IMF5 & IMF6 & IMF7 & Trend \\
\hline \multirow{2}{*}{ 1-step } & SVR-SP & 0.530 & 0.256 & 0.061 & 0.047 & 0.042 & 0.040 & 0.326 & 0.100 \\
& LassoCV & 0.594 & 0.178 & 0.033 & 0.002 & 0.001 & 0.000 & 0.000 & 0.000 \\
\hline \multirow{2}{*}{ 2-step } & SVR-SP & 0.670 & 0.407 & 0.198 & 0.067 & 0.041 & 0.042 & 0.327 & 0.100 \\
& LassoCV & 0.662 & 0.369 & 0.121 & 0.009 & 0.001 & 0.000 & 0.001 & 0.000 \\
\hline \multirow{2}{*}{ 3-step } & SVR-SP & 0.668 & 0.422 & 0.354 & 0.086 & 0.046 & 0.045 & 0.327 & 0.100 \\
& LassoCV & 0.663 & 0.401 & 0.262 & 0.023 & 0.002 & 0.001 & 0.001 & 0.000 \\
\hline \hline
\end{tabular}

1

2 


\section{Table $\mathbf{1 1}$ (on next page)}

The RMSE of VMD, EEMD and EMD at wind stations \#1 and \#2. 


\begin{tabular}{lllll}
\hline \hline \multirow{2}{*}{ Wind station } & Signal decomposition & \multicolumn{3}{c}{ RMSE } \\
\cline { 3 - 5 } & method & 1-step & 2-step & 3-step \\
\hline Site \#1 & VMD & 0.6395 & 0.6782 & 0.7793 \\
& EEMD & 0.6358 & 0.7301 & 0.8277 \\
& EMD (The proposed) & 0.5859 & 0.7531 & 0.8528 \\
\hline Site \#2 & VMD & 0.6664 & 0.6654 & 0.7111 \\
& EEMD & 0.5844 & 0.8404 & 0.8758 \\
& EMD (The proposed) & 0.5593 & 0.7540 & 0.7911 \\
\hline \hline
\end{tabular}

1 